phases: (1) Focus groups with key stakeholders, July- Nov 2019; (2) Establish special interest groups to develop resources, July 2020 onwards; (3) Disseminate information locally and nationally, May 2021 onwards.

Results The focus groups identified three core capabilities: (1) Assessment of frailty and palliative care needs, (2) Recognition of deterioration and symptom management, (3) Communication around advance care planning (ACP). We established six special interest groups related to frailty: recognition; resource mapping; partnerships; rehabilitation in care homes; dementia; and ACP.

Activities to date include: implementing frailty identification as routine practice across the hospice including adaption of electronic systems to plan and audit care, creating proformas for multidisciplinary meetings with GPs and DNs focusing on frailty; devising crib cards for ACP conversations using $\mathrm{CLaD}$ intervention methodology and management of frailty related symptoms e.g. delirium and building capability with local partners in rehabilitation and recognition of palliative care need. Early evaluation suggests wide spread adoption and increased confidence and understanding of frailty.

Conclusion We have shown that the palliative care needs of older people with frailty can be enhanced by highlighting information and resources. We plan to cascade further through local and national conferences.

\section{P-15 THE ROLE OF PROJECT ECHO IN BUILDING COMMUNITIES OF PRACTICE TO SUPPORT MENTAL HEALTH AND WELLBEING}

${ }^{1}$ Ruth Gray, ${ }^{2}$ Chris Jenkins, ${ }^{3}$ Helen McNally, ${ }^{2}$ Tracey McTernaghan. ${ }^{1}$ SE Health and Social Care Trust, Belfast, UK; ${ }^{2}$ Hospice UK, Belfast, UK; ${ }^{3}$ Ulster University, Newtownabbey, UK

10.1136/spcare-2021-Hospice.36

Background People living in prison may experience poor mental health, psychological disorders, and frequently have experienced traumatic episodes as both children and adults. As a post-conflict setting with high levels of Post-traumatic stress disorder in the general population, these challenges are likely particularly evident within Northern Ireland's prison population.

Aim(s) The Northern Ireland Prison Service within the Department of Justice is responsible for delivering prison services, while the South Eastern Health and Social Care Trust is responsible for delivering health care in the region's prisons. This study sought to assess whether Project ECHO is a useful tool for building communities of practice across agencies to address challenges in supporting mental health and wellbeing services within Northern Ireland's prisons.

Methods Project ECHO (Extension for Community Healthcare Outcomes) is a tele-mentoring programme that uses video-conferencing technology to deliver evidence-based, best practice guidance and education; and case-based learning through presentations by network participants. Interviews were conducted with members of the Northern Ireland Prisons ECHO network on the impact of the programme, and analysed thematically.

Results The ECHO approach was considered effective by participants in bridging knowledge gaps between different levels, service providers, and institutions involved in delivering mental health services in prisons. Wider changes in service delivery, catalysed through the relationships developed within ECHO, were described as encouraging a greater focus on client-centred planning. The ECHO network helped to catalyse other important changes within the prison service such as the development of a new referral pathway between the Northern Ireland Prison Service and the South Eastern Health and Social Care mental health team, and new operating procedures for keeping people safe in custody.

Conclusions This study show the value of digital-based solutions such as Project ECHO to build communities of practice between different institutions to achieve goals such as improving mental health and wellbeing in a complex prison population.

\section{P-16 SOCIAL PRESCRIBING AT END OF LIFE WITH A FOCUS ON THOSE WHO DO NOT TRADITIONALLY ACCESS HOSPICE CARE}

${ }^{1}$ Nicola Button, ${ }^{1}$ Maureen Hanley, ${ }^{2}$ Louise Willsher, ${ }^{3}$ Lisa Andrews, ${ }^{1}$ Lisa Parrish. ${ }^{1}$ St Helena Hospice, Colchester, UK; ${ }^{2}$ C360, Colchester, UK; ${ }^{3}$ CVST, Clacton, UK

\subsection{6/spcare-2021-Hospice.37}

In collaboration with both local Community Voluntary Services (CVS), Community 360, Community Voluntary Services Tendring and Essex Faith Covenant, St Helena Hospice has been successful in receiving grant funding from the Masonic Charitable Foundation for an exciting new project. This has further been supported financially by our Alliance End of Life Board.

In this Social Prescribing ${ }^{\mathrm{TM}}$ pilot project, we will recruit two additional engagement officers, one in both Colchester and Tendring to work with under-represented Black, Asian and minority ethnic and deprived groups, and any other communities who do not traditionally access hospice care. We will work to better understand their needs and barriers to accessing services. The Engagement Officers will be given training on social prescribing and end-of-life care and will work across CVSs and the hospice.

Our dashboard shows inequity of access for those living in areas of high deprivation and this project will link with our well established SafeHarbour project.

The Engagement Officers will work with existing services in the community to develop an awareness of the needs of these communities and propose modifications which would promote greater equality of access as well as supporting these communities to feel better informed at supporting their citizens at end-of-life. If gaps are identified within local community assets we will work with partners to develop, support and where appropriate seek investment in community assets. This collaborative approach would enable communities to co-produce solutions to improve health outcomes, with the Engagement Officer serving as a facilitator across community networks.

We hope community leaders will gain a better knowledge of what happens at end-of-life and feel empowered to shape the services available. Equally end-of-life providers would learn more about the community services available to support their patients and how our services need to be adapted in order to ensure equality of access for all. 